\title{
Complementary Sensory and Associative Microcircuitry in Primary Olfactory Cortex
}

\author{
Hauke F. Wiegand, ${ }^{1,4}$ Prateep Beed, ${ }^{1}$ Michael H. K. Bendels, ${ }^{2}$ Christian Leibold, ${ }^{5,6}$ Dietmar Schmitz, ${ }^{1,3,7 *}$ \\ and Friedrich W. Johenning ${ }^{1 \star}$ \\ ${ }^{1}$ Neuroscience Research Center, ${ }^{2}$ Neurophysics Group, Department of Neurology, ${ }^{3}$ Cluster of Excellence "NeuroCure," and ${ }^{4}$ Department of Psychiatry, \\ Charité-Universtiätsmedizin Berlin, 10117 Berlin, Germany, ${ }^{5 B}$ Bernstein Center for Computational Neuroscience Munich, 82152 Planegg-Martinsried, \\ Germany, ${ }^{6}$ Division of Neurobiology, Ludwig-Maximilians-Universität München, 82152 Planegg-Martinsried, Germany, and ${ }^{7}$ Bernstein Center for \\ Computational Neuroscience Berlin, 10115 Berlin, Germany
}

The three-layered primary olfactory (piriform) cortex is the largest component of the olfactory cortex. Sensory and intracortical inputs converge on principal cells in the anterior piriform cortex $(\mathrm{aPC})$. We characterize organization principles of the sensory and intracortical microcircuitry of layer II and III principal cells in acute slices of rat aPC using laser-scanning photostimulation and fast two-photon population $\mathrm{Ca}^{2+}$ imaging. Layer II and III principal cells are set up on a superficial-to-deep vertical axis. We found that the position on this axis correlates with input resistance and bursting behavior. These parameters scale with distinct patterns of incorporation into sensory and associative microcircuits, resulting in a converse gradient of sensory and intracortical inputs. In layer II, sensory circuits dominate superficial cells, whereas incorporation in intracortical circuits increases with depth. Layer III pyramidal cells receive more intracortical inputs than layer II pyramidal cells, but with an asymmetric dorsal offset. This microcircuit organization results in a diverse hybrid feedforward/recurrent network of neurons integrating varying ratios of intracortical and sensory input depending on a cell's position on the superficial-to-deep vertical axis. Since burstiness of spiking correlates with both the cell's location on this axis and its incorporation in intracortical microcircuitry, the neuronal output mode may encode a given cell's involvement in sensory versus associative processing.

\section{Introduction}

The piriform cortex (PC) is a three-layered palaeocortex and the largest component of the olfactory cortex. It receives direct sensory input from mitral cells in the olfactory bulb via the lateral olfactory tract (LOT) terminating in the outermost layer Ia. In addition to receiving sensory input in layer Ia, PC principal neurons receive both local and long-range intracortical connections in layers Ib, II, and III (Neville and Haberly, 2004; Wilson and Barkai, 2010). Current descriptions of PC imply a convergence of sensory and intracortical inputs onto principal cells in layers II and III. In this respect, the three-layered palaeocortex is different from the six-layered neocortex in sensory areas. In the neocortex,

Received Jan. 18, 2011; revised June 14, 2011; accepted July 7, 2011.

Author contributions:H.F.W., D.S., and F.W.J. designed research;H.F.W. and F.W.J. performed research; M.H.K.B. contributed unpublished reagents/analytic tools; H.F.W., P.B., M.H.K.B., C.L., and F.W.J. analyzed data; H.F.W., C.L., D.S., and F.W.J. wrote the paper.

${ }^{*} D$.S. and F.W.J. contributed equally to this work.

This work was supported by Deutsche Forschungsgemeinschaft Grants Exc 257, SCHM 1383/6-1, SFB 618, and SFB 665; and by the Bundesministerium für Bildung und Forschung (Bernstein Fokus, 01GQ0440, BCCN Berlin, Munich, 01GQ BFNL). We thank James Poulet for critically reading this manuscript and Susanne Rieckmann and Anke Schönherr for excellent technical assistance.

M.H.K.B. is a CEO of Morgentau solutions, a company selling a mapping software based on the one used in this publication. However, this paper was generated with a previous software version developed for research purposes.

Correspondence should be addressed to either Dietmar Schmitz or Friedrich W. Johenning, Neurowissenschaftliches Forschungszentrum, Charité-Universitätsmedizin Berlin, Charitéplatz 1, 10117 Berlin, Germany, E-mail: dietmar.schmitz@charite.de or friedrich.johenning@charite.de.

DOI:10.1523/JNEUROSCI.0285-11.2011

Copyright $\odot 2011$ the authors $\quad 0270-6474 / 11 / 3112149-10 \$ 15.00 / 0$ sensory and intracortical microcircuits are distributed to two cell populations in different layers. Sensory inputs from the thalamus target layer 4 . Layer 4 subsequently projects onto layer II/III, which distributes and receives intracortical associative fibers (De Felipe and Jones, 2010).

From the network theory perspective, different elements of microcircuit organization underlie sensory and associative memory-related cortical processing: afferent feedforward excitation in sensory microcircuits from relays such as the thalamus (or olfactory bulb) supports sensory (serial) processing. Recurrent feedback excitation in intracortical microcircuits mainly mediates associative memory-related processing in associative brain areas such as the CA3 region of the hippocampus [parallel processing (Treves and Rolls, 1992; Douglas and Martin, 2010)]. Both concepts underlie opposing theoretical network models of the PC (Ambros-Ingerson et al., 1990; Barkai et al., 1994). More recently, these concepts have been merged into the proposition of the anterior piriform cortex (aPC) as a "hybrid recurrent/feedforward pattern correlation network" (Haberly, 2001). In vivo current source density analysis in the aPC revealed the sequential activation of sensory and intracortical synapses on aPC principal cells (Ketchum and Haberly, 1993).

We aimed at a quantitative assessment of functional microcircuit organization in acute slices of rat aPC with respect to these two subcircuits (sensory and intracortical). We used fast two-photon population $\mathrm{Ca}^{2+}$ imaging to probe LOT-mediated afferent sensory inputs to layer II and laser-scanning photostimulation (LSPS) to 
quantify intracortical circuitry in layer II/III principal cells of the aPC. Comparing superficial and deep populations, cells in layer II are organized to receive decreasing sensory excitation from the olfactory bulb. Conversely, we observed a superficial to deep increase in intracortical inputs within layer II and from layer II to layer III. Canonical descriptions of cortical circuitry are based on homogenous layers of cells with distinct roles in sensory feature extraction, intracortical processing, or a mixture of both (Douglas and Martin, 2010). In the olfactory cortex, we demonstrate a more diverse principle of network organization: along the superficial-to-deep axis, aPC principal neurons integrate varying ratios of intracortical and sensory input by using converse gradients of sensory and associative microcircuitry. Furthermore, the position along this axis correlates with intrinsic cellular parameters that distinctly shape neuronal response properties.

\section{Materials and Methods}

Slice preparation. Experiments followed approved national and institutional guidelines for animal use. Acute slices of the aPC (400 $\mu \mathrm{m}$ thickness) were prepared from Wistar rats of either sex (age, postnatal days $18-25)$ in a coronal plane perpendicular to and containing the LOT (Johenning et al., 2009).

Electrophysiology. Whole-cell voltage- and current-clamp recordings were performed with a Multiclamp 700B Amplifier (Molecular Devices). Data were digitized (BNC-2090; National Instruments) at $5 \mathrm{kHz}$, lowpass filtered at $2 \mathrm{kHz}$, and recorded stimulation point specific with custom-made software. For calibration experiments, patch electrodes (with electrode resistances ranging from 3 to $6 \mathrm{M} \Omega$ ) were filled with (in mM) $135 \mathrm{~K}$-gluconate, $20 \mathrm{KCl}, 2 \mathrm{MgATP}, 10 \mathrm{HEPES}, 0.2 \mathrm{EGTA}, 5$ phosphocreatine, and $0.2 \%$ biocytin, $\mathrm{pH}$ 7.3. For mapping experiments, the intracellular solution consisted of (in mM) $150 \mathrm{~K}$-gluconate, $0.5 \mathrm{MgCl}_{2}$, 1.1 EGTA, 10 phosphocreatine, and $0.2 \%$ biocytin, $\mathrm{pH}$ 7.2. Cells were held in voltage clamp between -65 and $-75 \mathrm{mV}$. Initial access resistances were below $25 \mathrm{M} \Omega$ after breakthrough and not allowed to vary $>30 \%$ during the course of the experiment in the voltage-clamp mode. No access resistance compensation was used.

Glutamate uncaging and scanning of glutamate-evoked activity. The setup, materials, and methods for photolysis of caged glutamate have been described previously (Bendels et al., 2008; Beed et al., 2010). In brief, $25 \mathrm{ml}$ of ASCF containing $200 \mu \mathrm{M}$ MNI-caged L-glutamate (Tocris) was recirculated at $3-5 \mathrm{ml} / \mathrm{min}$. For uncaging, we used a modified pulsed UV laser at $355 \mathrm{~nm}$ (Rapp Optoelectronics). The optical system was adapted to achieve an effective light spot diameter of $15 \mu \mathrm{m}$ in the focal plane. The focal depth was kept constant at $50 \mu \mathrm{m}$ below the slice.

Histological procedures. Slices with biocytin-filled cells were fixed in 0.1

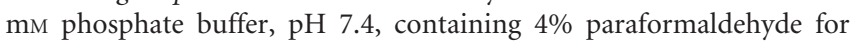
$24-48 \mathrm{~h}$. Staining and reconstruction were performed as described previously (Beed et al., 2010).

Two-photon $\mathrm{Ca}^{2+}$ imaging. For fluometric $\mathrm{Ca}^{2+}$ measurements, we modified the multicell bolus-loading procedure (Stosiek et al., 2003) adapted to slice preparations (Johenning and Holthoff, 2007). In brief, $200 \mu \mathrm{M}$ of the red morphological marker CellTracker Red CMTPX was added to $1 \mathrm{~mm}$ OG-BAPTA1-AM. Application was performed at three different locations (between layers I and II, in layer II, and between layers II and III) at 100 mbar. The application pipette was inserted dorsally from the LOT and the imaged field of view (toward the perirhinal fissure). The stimulation electrode was placed in layer 1a, ventrally between the LOT and the imaged field of view (i.e., toward the LOT). Intracortical inputs were selectively blocked by $30 \mu \mathrm{M}$ baclofen (Tang and Hasselmo, 1994; Franks and Isaacson, 2005; Johenning et al., 2009). Stimulation strength was adjusted to be at the threshold for activation of at least one cell in the population with two stimuli.

Two-photon imaging was performed on a two-photon laser-scanning system (Femto2D; Femtonics) equipped with a femtosecond laser tuned to $810 \mathrm{~nm}$ (Chameleon; Coherent). For epifluorescence, we used an Olympus XLUMPLFL $(20 \times / 0.95$ NA) water-immersion objective; trans-fluorescence and transmitted IR were collected with an Olympus oil-immersion condenser. A diagram of the light path can be obtained from Chiovini et al. (2010). Multiple cells (20-30) within a field of view were scanned at constant speed $(125$ or $250 \mathrm{~Hz})$ while intermediate sections were jumped over within $60 \mu \mathrm{s}$, using a spline-interpolated path (multiple line scanning) (Lorincz et al., 2007). Measurement control, data acquisition, and analysis were performed using the Matlab-based MES program package (Femtonics). Cells covered by the multiple line scan were blindly and randomly picked before stimulation.

Analysis and statistics. For cell-type classification, we used three parameters: input resistance, burst index, and normalized depth for layer II cells. The burst index was calculated as $\Delta t_{2} / \Delta t_{1}$, where $t_{1}$ is the time interval between the first and second action potentials (APs) and $t_{2}$ is the time interval between the second and third APs, in a 600-ms-long train of eight APs (Suzuki and Bekkers, 2006). The superficial and deep borders of layer II were defined on visual inspection of low-magnification differential interference contrast (DIC) images. Cells were localized on a normalized vertical axis, with 0 being the superficial border and 1 being the deep border.

For detection of synaptic events, we used the automatic detection method described by Bendels et al. (2008). Parameters used by the iterative algorithm were based on visual inspection of the raw data. Figure $3 B$ displays examples traces with events detected by the algorithm.

The algorithm used for discriminating photoactivated synaptic inputs (synaptic points) from random background activity is based on the observation that a presynaptic cell has several neighboring activation sites (see Fig. 2). Consequently, spatial correlation of inputs indicates specific activation. To extract significant spatial correlations, we tested the significance of the number of observed inputs in a restricted region against the null hypothesis of a random spatially uncorrelated pattern. A description of the algorithm used for the separation of specific events into synaptic points and background noise is given by Bendels et al. (2010).

To work out spatial differences in connectivity between the different cell populations, we defined a layer-specific connectivity coefficient, calculated as the ratio of positive synaptic points to all scanning points in a layer (synaptic points layer $_{\text {scanning points }}$ layer ).

For analyzing differences of input positions in relation to the dorsoventral main axis, we distinguished layer-specific dorsal and ventral sectors by constructing an axis through the cell body perpendicular to the surface or the aPC layer I/LOT border (see Figs. $3 E, F, 5 A$ ).

We observed a wide and patchy distribution of inputs (see, for example, Figs. 3, $E$ and $F$, and $5 A$ ). For each individual cell, we aimed at finding a measure of how the inputs are distributed on both sides of the axis. The first step was to calculate the sector-specific connectivity coefficient analogous to the layer-specific connectivity coefficient (synaptic points sector $_{\text {/ }}$ scanning points sector $)$, in which a sector is defined as the stimulated area on one side of the axis. A comparison of the relative weight of inputs on either side of the axis between different cells needs to be independent of the baseline connectivity. Therefore, it was necessary to correct for differences in overall connectivity. The correction factor for a cell's overall connectivity was determined as the quotient of scanning points TOTAL $_{\text {TA }}$ and

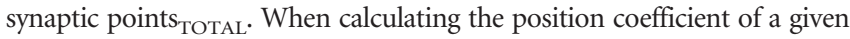
sector for a given cell (synaptic points SECTOR $_{\text {scanning points }}$ SECTOR $) \times$ (scanning points TOTAL $_{\text {synaptic points }}$ TOTAL), cells with large overall connectivity will have a small correction factor, and cells with small overall connectivity will have a large correction factor. We then subtracted the ventral from the dorsal position coefficient for each layer in every cell. Values $>0$ indicate an asymmetry toward the dorsal side, and values $<0$ indicate an asymmetry toward the ventral side.

For analysis of population $\mathrm{Ca}^{2+}$ imaging data, fluorescence traces are expressed as relative changes in fluorescence $(\Delta F / F=(F-F 0) / F 0)$, with $F 0$ being the averaged prestimulus fluorescence. Traces from five consecutive experiments were averaged. For comparison with the $125 \mathrm{~Hz}$ data, two consecutive frames were averaged in the data acquired with $250 \mathrm{~Hz}$, which leads to an effective sample rate of $125 \mathrm{~Hz}$. We determined the onset sweep number (at $125 \mathrm{~Hz}$ scanning speed, each $8 \mathrm{~ms}$ line cycle represents one sweep) of each individual cell's somatic $\mathrm{Ca}^{2+}$ transient. For determination of the signal onset, the SD of the baseline signal was calculated. The line scan data were binned in $8 \mathrm{~ms}$ sweeps based on the $125 \mathrm{~Hz}$ sampling frequency to compensate for differences in acquisition 

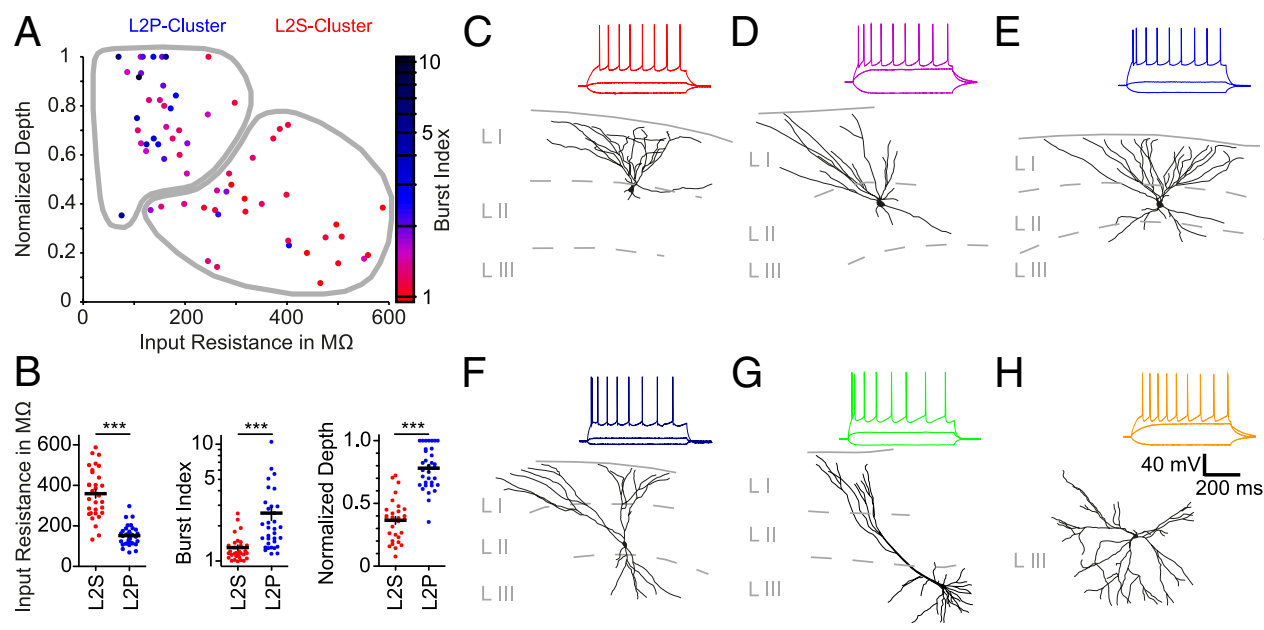

Figure 1. aPC principal cells are organized in a continuous gradient. $A$, Plot of input resistance versus depth in layer Il of the aPC. The color code refers to the burst index: red, low spike frequency adaptation; blue, high spike frequency adaptation. Within layer II, a functional and morphological diversity of different principal cells can be identified. Gray lines indicate populations defined for statistical comparisons between superficial and deep cells using a $k$-means cluster algorithm. In layer II, we distinguish two clusters for analysis: L2S containing more semilunar-like cells and L2P with more pyramidal-like cells. $\boldsymbol{B}$, Comparison of the two clusters for the parameters underlying the analysis yields significant differences. $C-G$, Biocytin reconstructions and input- output curves of cells demonstrating the vertical transition. C, "Canonical" semilunar cell (cluster L2S); $\mathbf{D}$, semilunar-pyramidal transition cell (cluster L2S); $\boldsymbol{E}$, superficial pyramidal cell (cluster L2P); $\boldsymbol{F}$, superficial to deep pyramidal transition cell (cluster L2P); G, deep pyramidal cell (L3P). $\boldsymbol{H}$, Biocytin reconstruction and input- output curve of a multipolar cell used for calibration (L3M).

time points between subsequent pixels in a scanned line. The onset was defined as the first sweep where the amplitude was larger than $2 \times \mathrm{SD}$ of the baseline $\left(\mathrm{SD}_{\mathrm{b}}\right)$. To qualify as an onset signal, this increase had to be followed by at least five sweeps with peak amplitudes larger than $2 \times \mathrm{SD}_{\mathrm{b}}$ interrupted by a maximum of six sweeps with peak maxima below $2 \times$ $\mathrm{SD}_{\mathrm{b}}$. These criteria were verified in control experiments; single APs were evoked by synaptic stimulation and recorded in cell-attached mode. The accompanying $\mathrm{Ca}^{2+}$ transient was measured in bolus-loaded layer II cells using image acquisition conditions similar to the ones used for collecting the population data. The single-AP-induced $\mathrm{Ca}^{2+}$ transients were clearly identified by the proposed detection criteria in five of five cells from independently stained slices (see Fig. 6A). Cells were excluded as compromised cells if there was a constant rise of the fluorescence signal for $200 \mathrm{~ms}$ after stimulus. Selection of cells for line scanning was done blindly before the first stimulus was given. Layer II was subdivided into cell rows blindly before analysis. Cells from each experiment were dichotomized into a superficial (superficial half of rows in an experiment) and a deep (deep half of rows in an experiment) group. If there was an uneven number of rows, the row in the middle was not analyzed (see Fig. $6 B$ ).

Statistical tests were performed using a two-tailed Mann-Whitney $U$ test and Kruskal-Wallis test with Dunn's multiple comparison as a post hoc test (referred to as Kruskal-Wallis test) as appropriate. Testing of correlations was based on Pearson's correlations. Numerical values are given as mean \pm SEM. The detection of synaptic events, the calibration analysis, and the cluster analysis were performed using Matlab (The Mathworks); all other statistical and graphical analysis was performed with standard software.

\section{Results}

\section{Distinction between principal cell types in the aPC}

Based on different projection patterns, the compact layer II has been subdivided into a superficial layer IIA and a deep layer IIB (Haberly and Price, 1978). Within layer II, there is a functional and morphological diversity of different principal cells. At the extreme, pyramidal cells deep in layer II (L2Ps) have been morphologically (Haberly, 1983), biophysically (Suzuki and Bekkers, 2006, 2011), and functionally (Suzuki and Bekkers, 2011) distinguished from semilunar cells (L2Ss) that are located more superficially. In contrast to this apparent dichotomy, a graded morphological transition from semilunar cells to superficial pyramidal cells in layer II has been described, yielding semilunar-pyramidal transition cells (Yang et al., 2004). Superficial pyramidal cells in layer II are gradually replaced by layer III pyramidal cells (L3Ps) constituting the main population of principal neurons in superficial layer III. In deep layer III, multipolar cells (L3Ms) dominate as the main principal cell type (Neville and Haberly, 2004).

Different principal cell types in the aPC were distinguished based on characteristic biophysical properties (Suzuki and Bekkers, 2006), vertical position, and morphology (Haberly, 1983; Yang et al., 2004), although the latter was only taken into account when probing the excitability of cells after laser stimulation. We analyzed excitatory cells distributed over the whole vertical axis of layer II. When plotting input resistance against normalized position on the vertical axis of layer II, we observed a linearly correlated transition from superficial cells with high input resistance to deep cells with low input resistance (Fig. $1 A ; r=-0.67 ; p<$ $0.001 ; n=64$; see Materials and Methods for details). The burst index (indicating a cell's rate of spike frequency adaptation; see Materials and Methods) was also correlated with the input resistance in layer II cells (Fig. $1 A ; r=-0.44 ; p<0.001 ; n=64$ ). High burst indices (i.e., a high rate of spike frequency adaptation correlated with an initial high-frequency burst) occur in deep cells with low input resistance, and low burst indices occur in superficial cells with high input resistance.

To facilitate subsequent statistical analysis of local intracortical microcircuitry in superficial and deep cells of layer II, cells in this semilunar- to pyramidal-like transition were clustered into two groups. Taking into account three parameters, input resistance, burst index, and normalized vertical depth in layer II, we grouped the cells by a $k$-means cluster analysis (repeated with 100 different initial conditions). Based on these three parameters, we distinguished two spatially overlapping clusters (L2S and L2P cluster; Fig. 1A). Within those clusters, we observed highly significant differences between L2Ss and L2Ps for all three parameters (input resistance, $p<0.001$; burst index, $p<0.001$; position, $p<0.001$; Kruskal-Wallis test; Fig. $1 B$ ).

We note that here cluster analysis was merely used to generate groups for statistical comparison of intracortical connectivity. 
Although clustering (necessarily) assumes discrete cell populations, it is important to stress that our data cannot be interpreted to validate (nor to disprove) the existence of the two distinct cell clusters established by Suzuki and Bekkers (2011) using a much larger number of phenotypical indicators. Alternatively, gradients observed in our data would also be compatible with a continuous transition from a semilunarto a superficial pyramidal-like phenotype within layer II (see Discussion).

In layer III, we could morphologically differentiate L3Ps and L3Ms. L3Ps have an early onset of AP firing after depolarization, an initial burst when firing more than two APs (high burst index; i.e., a high rate of spike frequency adaptation), and a low input resistance and are found in superficial layer III. Multipolar cells demonstrate a long latency of AP firing after depolarization, a regular firing pattern, and a low input resistance similar to L3P and they are found in deep layer III (Fig. $1 G, H)$.

\section{Calibration of spatial resolution}

We next wanted to compare the layerspecific intracortical connectivity patterns between L2Ss, L2Ps, and L3Ps using LSPS. Focal photolysis of caged glutamate by LSPS induces direct and indirect synaptic responses in the recorded neuron (called a target cell). The direct responses are evoked by glutamate uncaged close to the somatodendritic compartment of the recorded target cell. Indirect synaptic responses reflect suprathreshold direct activation, i.e., AP firing of a presynaptic neuron projecting onto the recorded neuron (called a source cell). We first determined the laser intensity that permits maximal spatial resolution. A measure of spatial resolution for LSPS is the critical distance $d^{*}$, which is the distance from the putative source cell's soma where $75 \%$ of all cumulated APs could be evoked as direct responses. The distance $d^{*}$ depends on cell type and laser intensity. It estimates the distance between cell soma and dendritic hot spots, i.e., the location on the dendritic arbor from which an AP is evoked by photolysis of caged glutamate (Shepherd et al., 2003; Bendels et al., 2008; Beed et al., 2010). We generated spatial profiles of AP firing of the main excitatory cells in all layers of the aPC in current clamp. We scanned a radius of $150 \mu \mathrm{m}$ for the superficial cells (Fig. $2 A$ ) or $300 \mu \mathrm{m}$ for the deep cells (Fig. $2 B, C$ ). The scanning pattern consisted of points with $30 \mu \mathrm{m}$ spacing. In Figure 2, we overlayed camera lucida reconstructions of representative cells with subthreshold (black) and suprathreshold (red) direct responses elicited at each scanning point. We observed perisomatic clustering of APs (Fig. $2 \mathrm{~A}-$ $C)$. Figure $2 D-F$ displays the fraction of APs depending on the distance from the cell soma for each cell type at the laser intensity used for our experiments. Calibration was performed for a wide range of laser intensities, and the laser intensity yielding the best resolution was used throughout the mapping experiments. The resulting cell-type-specific $d^{\star}$ values at the laser intensity used for our subsequent mapping experiments were as follows: $\mathrm{L} 2$ principal cells (L2Ps and L2Ss grouped as L2Cs, $n=26$ ), $83.5 \mu \mathrm{m}$; L3 9), $170.7 \mu \mathrm{m}$.
B

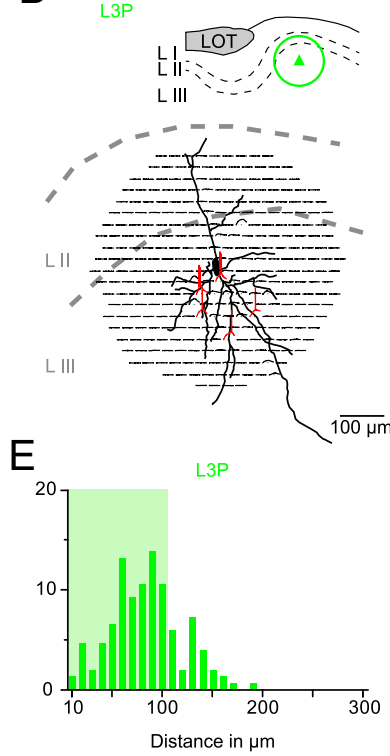

C
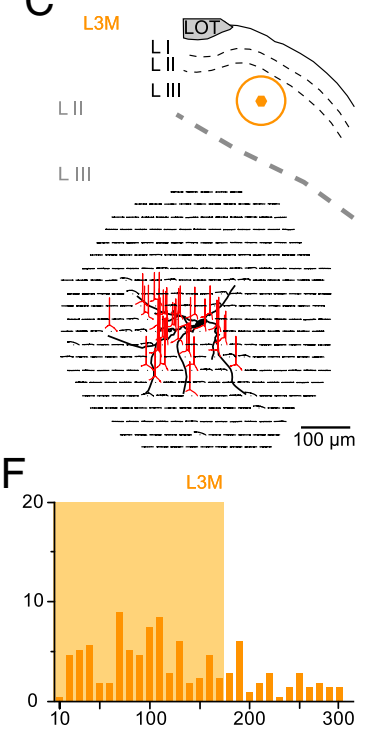

Figure 2. Resolution of photoactivation by glutamate uncaging. $\boldsymbol{A}-\boldsymbol{C}$, Sketches on top mark the position of three example principal cells within the aPC coronal slice. Below, biocytin reconstructions are overlayed with direct inputs evoked by LSPS fured in current clamp. Perisomatic suprathreshold activation is demonstrated by red APs. The stimulation pattern consisted calibrated cells' somata. APs were counted in $10 \mu \mathrm{m}$ spatial bins. Shaded boxes correspond to $d^{*}$, where $75 \%$ of all inputs were observed. L2Ps and L2Ss grouped as L2Cs $(n=26), 83.5 \mu \mathrm{m} ; \mathrm{L} 3$ pyramidal cell (L3P; $n=6), 97.2 \mu \mathrm{m}$; multipolar cell (L3M; $n=$

pyramidal cell (L3P, $n=6$ ), $97.2 \mu \mathrm{m}$; multipolar cell (L3M, $n=$ 9), $170.7 \mu \mathrm{m}$ (Fig. 2D-F).

\section{Generation of principal cell-type-specific afferent input maps from different aPC layers}

For interpretation of afferent maps, we first had to define the time interval after the uncaging flash used for collecting indirect monosynaptic inputs. Synaptic inputs were collected up to 100 ms after UV photolysis (Fig. $3 A, B, G$, light blue boxes around the example traces). Representative traces of the time course of APs evoked by direct stimulation in calibration experiments are displayed in Figure $3 A$. The bursting behavior of superficial cells and the long membrane time constant of deep-layer principal cells (Tseng and Haberly, 1989) explain temporal delays in the range of $100 \mathrm{~ms}$ after the uncaging flash (see Fig. $3 D$ for a quantification of temporal delays to first and last APs as evoked by uncaging). This is reflected by the increase in PSC rates in this time frame (Fig. 3C).

Polysynaptic activation would result in degradation of spatial resolution of our data. We therefore analyzed our calibration data to see whether indirect inputs evoke suprathreshold postsynaptic responses resulting in AP firing. In L2Ps and L2Ss, APs within our $100 \mathrm{~ms}$ analysis window were always elicited by direct activation of the recorded cells (100\%, average of 26 cells). In L3Ps ( $n=6$ cells), the average probability of indirectly activated suprathreshold responses was $2.2 \pm 1 \%$ per cell. In L3Ms ( $n=9$ cells), this average probability value was $3.8 \pm 6 \%$. We therefore consider uncaging-induced synaptic inputs to be mostly monosynaptic.

We separated the direct and indirect synaptic responses by their different delay-to-onset times. Direct responses were elicited almost immediately in a time window of $10 \mathrm{~ms}$ after the UV 

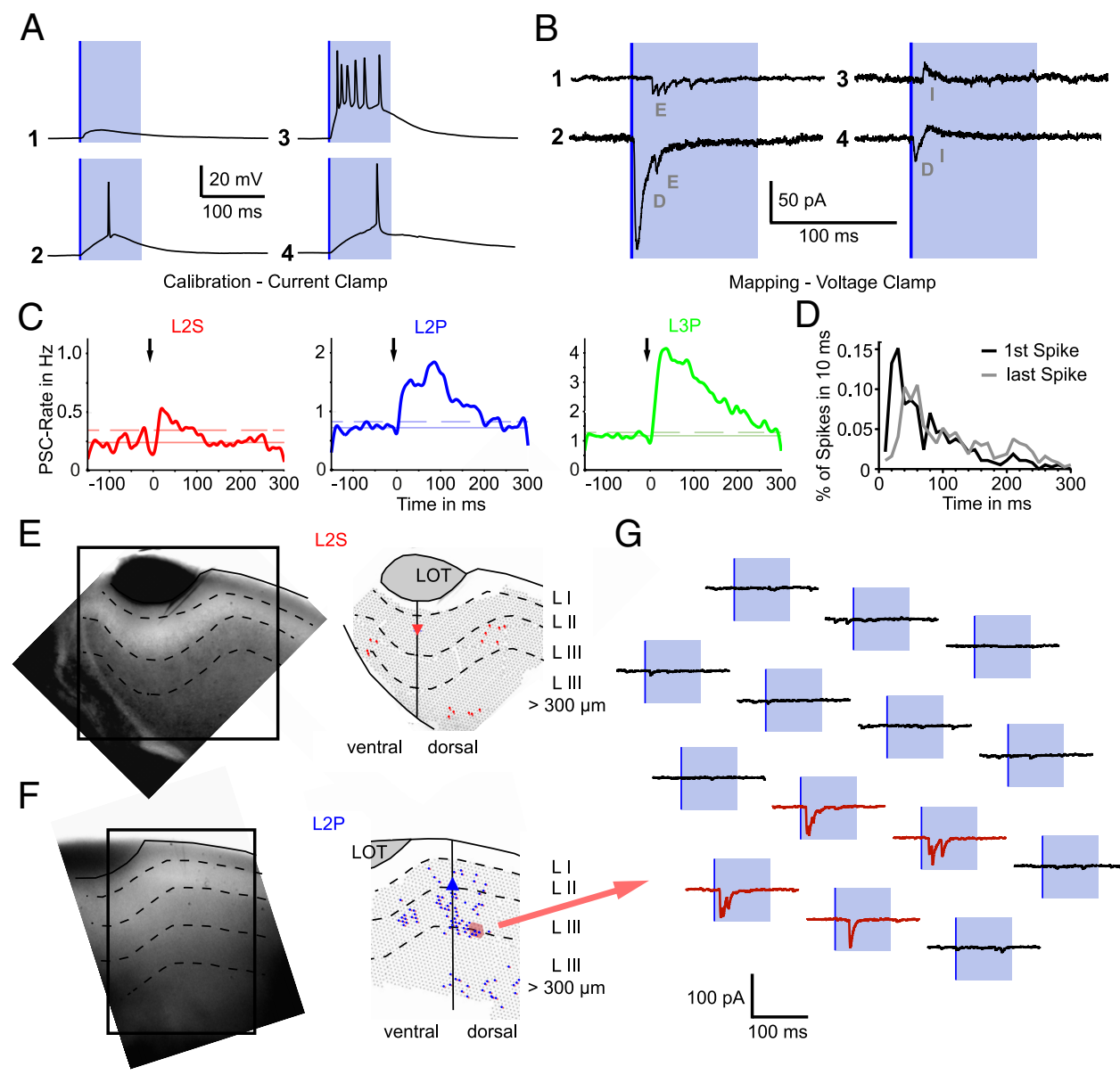

G

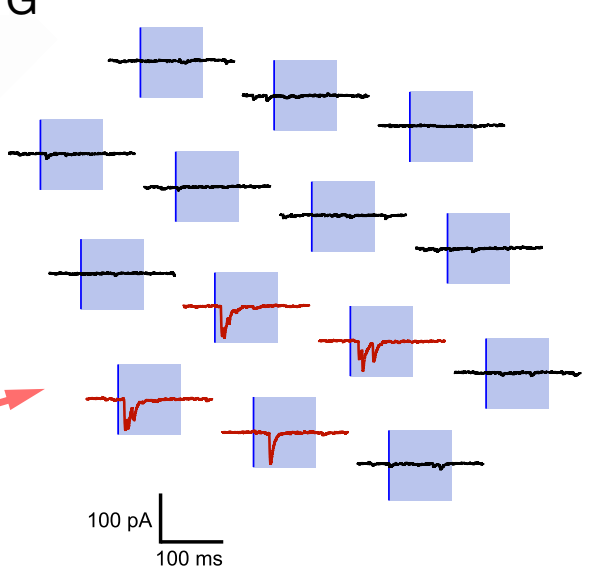

Figure 3. Mapping local recurrent feedback excitation. $A$, Representative traces displaying direct activation of neurons measured in current clamp. The UV flash is indicated by the dark blue line. The light blue shaded area indicates the $100 \mathrm{~ms}$ critical time interval analyzed for indirect inputs by the detection algorithm. $\boldsymbol{B}$, Representative traces displaying the different classes of events in voltage clamp detected by the analysis algorithm. E, Indirect excitatory response; D, direct excitatory response; I, indirect inhibitory response. C, Averaged PSC incidence rates for L2S (red), L2P (blue), and L3P (green) plotted over time. The arrows indicate the time point of photostimulation. A significant photostimulation-related increase can be observed in all cell types. $D$, Frequency distribution histogram of the latency of the first and last AP after the UV flash in $10 \mathrm{~ms}$ bins ( 370 traces with APs from 27 cells). $\boldsymbol{E}$, Example map of an L2S (red triangle, target cell). $F$, Example map of an L2P (blue triangle, target cell). Left, Low-magnification DIC images of the aPC coronal slice. Right, Corresponding scanning rasters and target cells are projected on the schematic drawings of the DIC images. Colored points indicate positive synaptic points detected as inputs from source cells. LI, layer I (not mapped); L II, layer II; L III $<300 \mu \mathrm{m}$, the first $300 \mu \mathrm{m}$ of layer III parallel to the layer II/layer III border; L III >300 $\mu \mathrm{m}$, the part of layer III below $300 \mu \mathrm{m}$. The raster consisted of stimulation points separated by $30 \mu \mathrm{m}$. G, Example traces from the stimulation raster (red in A2): $200 \mathrm{~ms}$ around the UV flash (indicated by dark blue line) are plotted. The light blue shaded area indicates the $100 \mathrm{~ms}$ critical time interval analyzed for indirect inputs by the detection algorithm. Traces positive for intracortical inputs are plotted in red, and negative traces are plotted in black.

flash (flash indicated as dark blue stripe in example traces in Fig. 3, $A$, $B$, and $G$; see also Materials and Methods). Indirect activation can be extracted on top of direct activation (Fig. $3 B$, trace 2), enabling us to also define local synaptic points. All inward currents are defined as EPSCs and could be clearly distinguished from the unanalyzed inhibitory outward currents (Fig. 3B, traces 3 and 4).

The synaptic responses collected in the specified time interval contain specific photo-induced synaptic inputs and unspecific spontaneous activity. The next step was the distinction between such photo-evoked and spontaneous responses. For discrimination between specific synaptic inputs and background activity, we used a spatial correlation-based algorithm to extract presynaptic input locations (see Materials and Methods; Bendels et al., 2010; Beed et al., 2010). Scanning points corresponding to photoactivation-induced inputs were termed synaptic points, and afferent maps were constructed based on these synaptic points [red points in Fig. $3 E(\mathrm{~L} 2 \mathrm{~S})$, blue points in Fig. $3 F(\mathrm{~L} 2 \mathrm{P})$, and green points in Fig. $5 A(\mathrm{~L} 3 \mathrm{P})]$.
Input from distinct layers was compared for different principal cells: mapping of local intracortical synaptic inputs was performed for L2Ss, L2Ps, and L3Ps. For mapping, a hexagonal grid with a point-to-point distance of $30 \mu \mathrm{m}$ was projected across the different layers (Figs. $3 E, F, 5 A$ ). For analysis, the scanning region was grouped into the following aPC cortical layers: layer II (based on the spatial resolution calculated from our calibration data, we did not subdivide layer II into a deep and superficial layer for the source-cell analysis), superficial layer III (first $300 \mu \mathrm{m}$ parallel to the layer II-layer III border), and deep layer III (below superficial layer III; Figs. $3 E, F, 5 A$ ).

\section{L2Ss, L2Ps, and L3Ps: comparison of intracortical connectivity}

For statistical comparison, target cells were classified as follows. Layer II cells were grouped according to the aforementioned cluster analysis (superficial layer II "semilunar phenotype" L2Ss vs 
deep layer II "pyramidal phenotype" L2Ps; Fig. 1A). L3Ps were identified as indicated above.

As a result of anatomical variances between acute brain slice preparations, the number of scanning points per layer sampled in individual experiments differs. To compare connectivity between different cell types (i.e., across slices and experiments), it was necessary to normalize the number of detected synaptic points per layer to the total number of scanned points in this layer. For this purpose, we calculated layer-specific connectivity coefficients for each cell (synaptic points $_{\text {layer }} /$ scanning points $_{\text {layer }} ;$ Figs. $3 E, F$, $5 A)$. Target cell-type-specific microcircuitry organization of the aPC can be evaluated by comparing layer-specific connectivity coefficients between cell types. A large connectivity coefficient indicates strong intracortical connections to a recorded target neuron from a given stimulated layer.

Layer II is the cell layer containing most of the aPC principal cells (Neville and Haberly, 2004). From layer II, we observe a significantly higher connectivity coefficient in L2Ps $(n=13)$ and L3Ps $(n=15)$ compared with L2Ss $(n=19$; L2P vs L2S, $p<0.001$; L3P vs L2S, $p<0.05$; Fig. $4 A$ ).

L3Ps received significantly more inputs from deep and superficial layer III than L2Ss [superficial layer III L3P ( $n=$ $14)$ vs L2S, $p<0.01$; deep layer III L3P $(n=17)$ vs L2S, $p<0.01$ ] (Figure $4 A)$. Comparing L2Ps to L2Ss, the intracortical connectivity from superficial and deep layer III was not significantly different [superficial layer III inputs: L2P $(n=13)$ vs L2S $(n=15)$, n.s.; deep layer III inputs: L2P $(n=14)$ vs L2S $(n=15)$, n.s.; Fig. $4 A]$.

Next, we compared intracortical connectivity between L3Ps and L2Ps. For stimulation sites in layer II and superficial layer III, the average recurrent feedback connectivity of L2Ps was not significantly different from L3Ps (Fig. 4A). For stimulation sites in deep layer III, we observed a significantly higher connectivity coefficient for L3Ps than for L2Ps $(p<0.01$, Kruskal-Wallis test used for all statistical comparisons in Fig. 4A). In summary, based on the groups of principal cells defined by clustering in layer II, we observe a superficial to deep increase in the strength of an aPC principal cell's intracortical connectivity with respect to the target cell's position on the vertical axis (L2S $<$ L2P $<$ L3P).

We then plotted the input resistance (as a parameter indicative for a more L2S-like or a more L2P/L3P-like phenotype) of the mapped cells against their connectivity coefficient. Overall, intracortical connectivity from all cortical layers clearly depended on input resistance. Low input resistances were associated with highly connected cells (Fig. $4 B ; r=-0.49$; $p<0.001 ; n=53$ ). We found a similar relationship between L2Ss, L2Ps, and L3Ps when plotting the overall intracortical connectivity against burst index (Fig. $4 C ; r=0.66 ; p<0.001 ; n=53$ ). The distribution of data points in these correlation plots suggests scaling of func-
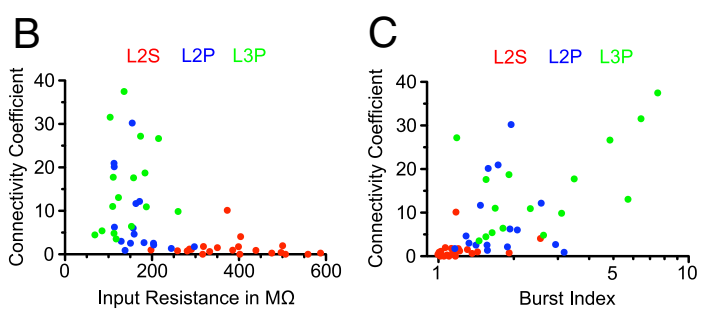

Figure 4. Intracortical connectivity increases from superficial to deep. $\boldsymbol{A}$, Statistical comparison of the connectivity coefficient of L2S, L2P (as distinguished by the cluster analysis), and L3P. From layer II, L2Ps $(7.3 \pm 2.2, n=13)$ and L3Ps $(7.7 \pm 2.5, n=15)$ display a significantly higher connectivity coefficient than L2Ss $(1.0 \pm 0.3, n=19 ; \mathrm{L} 2 \mathrm{P}$ vs L2S, $p<0.001 ; \mathrm{L} 3 \mathrm{P}$ vs $\mathrm{L} 2 \mathrm{~S}, p<0.05)$. 13. $2 S .12$ S both superficial and deep layer III, L3Ps were significantly higher connected than L2Ss (superficial layer III, L3Ps: 14.4 $\pm 4.3, n=$ 14; deep layer III, L2Ps: $15.6 \pm 2.8, n=17$; superficial layer III, L3P vs L2S: $p<0.01$; deep layer III, L3P vs L2S: $p<0.01$ ). From layer II and superficial layer III, the average intracortical connectivity of L2Ps was not significantly different compared with target cell's input resistance ( $r=-0.49 ; p<0.001 ; n=53)$. C, Overall connectivity from all layers correlates with the
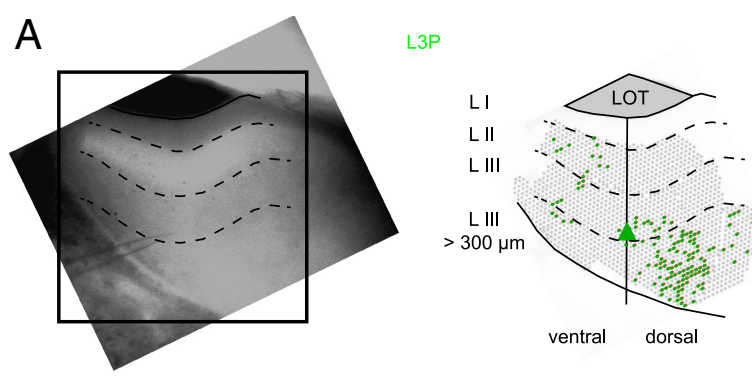

B

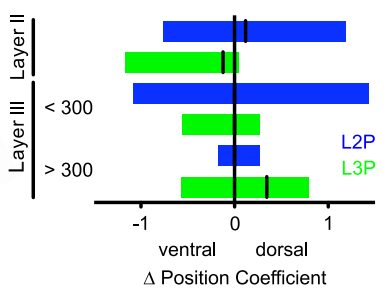

Figure 5. L3Ps receive asymmetric inputs on the ventrodorsal axis. $\boldsymbol{A}$, Left, DIC image of the aPC superimposed with the layering 作 作 705 for deep layer III vs layer II inputs onto L2Ps, Mann-Whitney U test]. L3Ps have a clearly asymmetric position shift of their deep inputs toward the dorsal orientation [layer II median $\Delta,-0.12(n=14)$; superficial layer III $\Delta, 0(n=16)$; deep layer III median $\Delta,-0.34$ ( $n=18$ ); $p<0.05$ for deep layer III vs layer II inputs onto L3Ps, Mann-Whitney $U$ test].

tional microcircuit incorporation with the intrinsic cellular properties input resistance and spike frequency adaptation along the superficial-to-deep axis.

\section{Layer III pyramidal cells: dorsoventral asymmetry of recurrent feedback excitation}

So far, we quantified a given cell's incorporation into the local intracortical microcircuitry. We next asked whether the inputs were distributed symmetrically on the dorsoventral axis preserved in our coronal slicing plane. To analyze symmetry on the dorsoventral axis, we constructed each cell's main axis (the axis perpendicular to the layer I surface/LOT border that intersected the cell body; Figs. $3 E, F, 5 B$; see also Beed et al., 2010) to divide the inputs into a ventral and a dorsal sector (Figs. $3 E, F, 5 A$ ). To prevent the overrepresentation of cells with a high connectivity coefficient in our analysis, we corrected for a given cell's overall connectivity, resulting in a layer- and sector-specific position coefficient (for details, see Materials and Methods). This way, we could directly compare the relative weight of synaptic inputs from the dorsal and ventral sector as defined by the main axis between cells. For measuring differences between cell types, we calculated the layer-specific difference $\Delta$ between the dorsal and ventral sector's position co- 
A
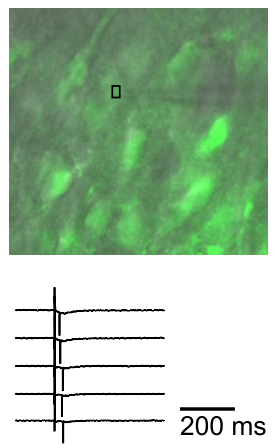

whd Mvmlw $\frac{0.2 \Delta \mathrm{F} / \mathrm{F}}{200 \mathrm{~ms}}$

B

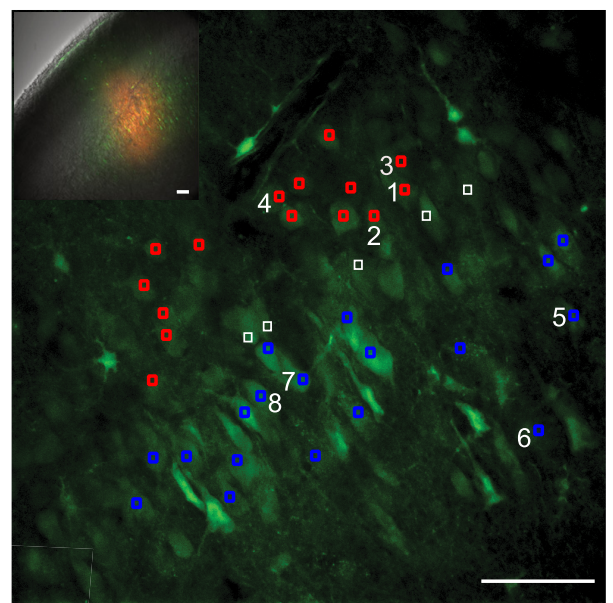

C

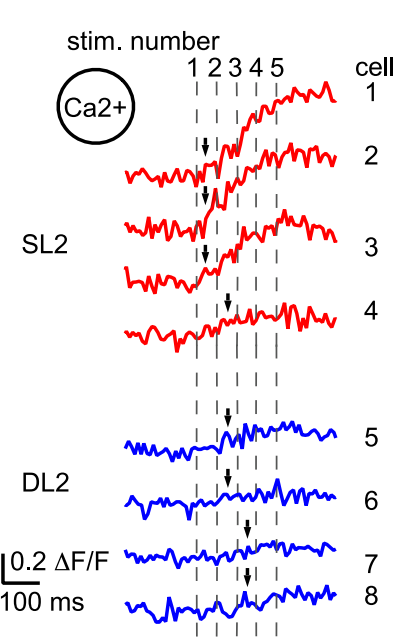

$\mathrm{D}$
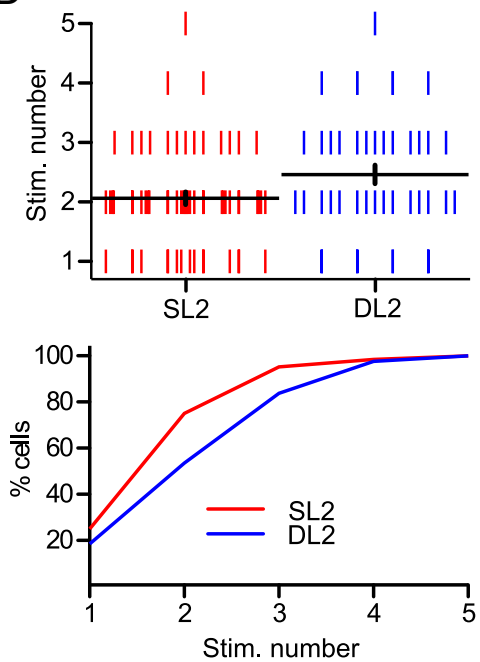

Figure 6. Single-cell excitability probing of sensory layer la inputs to deep and superficial layer II neurons. $\boldsymbol{A}$, Top, aPC bolus loaded with 0G-BAPTA1-AM. The overlay of green fluorescence and transmitted infrared to visualize a bolus-loaded cell and patch pipette are shown. The black box indicates position of cell used for simultaneous cell-attached recording and $\mathrm{Ca}^{2+}$ imaging. Middle, Five subsequent synaptically induced single APs measured in a cell-attached patch underlying the averaged $\mathrm{Ca}^{2+} \operatorname{tran}^{-}$ sient at the bottom. $\boldsymbol{B}$, Inset, Overlay of fluorescence and transmitted infrared (scale bars, $50 \mu \mathrm{m}$ ). The high-magnification image of layer II neurons is from the inset. Numbers 1-8 label example cells corresponding to the traces in $\boldsymbol{C}$. Blue boxes correspond to superficial layer II cells, white boxes correspond to cells neither attributable to superficial and deep half of rows (middle), and red boxes correspond to cells in deep half of layer II cell rows (scale bar, $50 \mu \mathrm{m}$ ). C, Averaged $\mathrm{Ca}^{2+}$ traces evoked by extracellular electrical stimulation of layer la (layer lb blocked by baclofen) inputs to superficial (red) and deep (blue) layer II cells as labeled in $\boldsymbol{B}$. The vertical gray lines correspond to the time points each of the five pulses were delivered with an interpulse interval of $50 \mathrm{~ms}$. Black arrows are placed in the stimulation interval in which the onset could be detected. $\boldsymbol{D}$, Top, 0 n average, superficial cells in layer II display the onset of averaged $\mathrm{Ca}^{2+}$ transients indicating AP firing after significantly less stimuli than deep cells in layer II [mean stimulus number (Stim. number), $2.1 \pm 0.1$ vs $2.5 \pm 0.16$ a.u., respectively; $p<0.05$, Mann-Whitney U test]. Bottom, Cumulative frequency of data displayed at the top.

efficient $\left(\Delta=\right.$ position coefficient $_{\text {dorsal }}-$ position coefficient $\left._{\text {ventral }}\right)$. Analyzing inputs from layer II $(n=14)$ and deep layer III, onto L2Ps, we found almost symmetric connectivities in the dorsal and the ventral sector relative to the main axis $(n=12 ; p>0.05$ for deep layer III vs layer II inputs onto L2Ps, Mann-Whitney $U$ test) (Fig. 5C). The L3Ps revealed an asymmetric spatial shift of their deep layer III inputs $(n=18)$ toward the dorsal direction when compared with layer II inputs $(n=14)$ on the same cell type $(p<0.05$ for deep layer III vs layer II inputs onto L3Ps, Mann-Whitney $U$ test; Fig. 5C). In summary, deep layer III intracortical inputs to L3Ps reveal an asymmetric organization along the dorsoventral axis.

Analysis of sensory input by fast twophoton population $\mathrm{Ca}^{2+}$ imaging reveals stronger recruitment of superficial layer II cells

Using current source density analysis in vivo, sensory synaptic activation has been demonstrated to precede intracortical synaptic activity in the aPC (Ketchum and Haberly, 1993). We pharmacologically isolated this early, sensory component of aPC processing by selectively blocking associative fibers with baclofen (Tang and Hasselmo, 1994; Franks and Isaacson, 2005; Johenning et al., 2009). To compare the relative weight of sensory afferents within simultaneously stimulated populations of superficial and deep cells in aPC layer II, we used the multiple line scan technique (Lorincz et al., 2007) for fast two-photon population $\mathrm{Ca}^{2+}$ imaging. Somatic $\mathrm{Ca}^{2+}$ transients evoked by synaptic stimulation indicate AP firing and scale with synaptic input strength (Smetters et al., 1999; Johenning and Holthoff, 2007; Dunfield and Haas, 2009, 2010). We first confirmed that our imaging approach faithfully reports single APs. We combined cell-attached recordings of layer II cells bolus loaded with OG-1-AM with $\mathrm{Ca}^{2+}$ imaging. We were able to detect single-AP-mediated $\mathrm{Ca}^{2+}$ signals in five of five cells from five independently loaded brain slices (Fig. 6A). Multiple line scanning thus enables us to measure single-AP-mediated somatic $\mathrm{Ca}^{2+}$ transients in populations of deep and superficial layer II cells simultaneously at a temporal resolution of $125 \mathrm{~Hz}$.

On the level of the aPC network, the timing of a cell's AP output in relation to the other cells is the critical factor. In vivo, global inhibition in the aPC permits only for small windows of opportunity for AP firing (Poo and Isaacson, 2009). Cells reaching the AP threshold too late will be silenced by inhibitory connections (Luna and Schoppa, 2008; Poo and Isaacson, 2009; Stokes and Isaacson, 2010; Suzuki and Bekkers, 2010). After a given sensory stimulus train, differences in onset times of the AP-induced somatic $\mathrm{Ca}^{2+}$ transient therefore reflect the degree of a given cell's recruitment by sensory afferents.

Fast population $\mathrm{Ca}^{2+}$ imaging at $125 \mathrm{~Hz}$ enabled us to identify the sequence of cells recruited in a train of synaptic stimulation (Fig. $6 B, C$ ). For a train of five layer 1 a stimuli at $20 \mathrm{~Hz}$, we analyzed the temporal sequence of AP firing onset in layer II cell populations after isolated sensory afferent stimulation (Fig. 6C). We first determined the onset of each individual cell's somatic $\mathrm{Ca}^{2+}$ transient (for details, see Materials and Methods). In the combined cell-attached and $\mathrm{Ca}^{2+}$-imaging experiments, single APmediated onsets of the $\mathrm{Ca}^{2+}$ transients were detected in a temporal window of 10-30 ms after delivery of the synaptic stimulus. By an- 
alyzing the onset time of the $\mathrm{Ca}^{2+}$ transient, we could estimate the stimulus number in a train after which each individual cell started to display a suprathreshold response. For further analysis, cells were categorized according to the position of the responding cells along the vertical axis yielding two groups: superficial layer II cells (SL2s; $n=64 / 5 / 3$ cells/slices/animals) and deep layer II cells (DL2s; $n=43 / 5 / 3$ cells/slices/animals; see Materials and Methods for further details).

On average, in $20 \mathrm{~Hz}$ trains of five extracellular stimuli, SL2 cells exhibited an onset of the AP-mediated somatic $\mathrm{Ca}^{2+}$ transient after significantly less stimuli than DL2 cells (mean stimulus number, $2.1 \pm 0.1$ vs $2.5 \pm 0.16 ; p<0.05$, Mann-Whitney $U$ test; Fig. $6 D$ ). AP firing in response to our specific train of sensory stimuli in aPC layer II cells thus occurs in a distinct temporal pattern: superficial layer II cells respond earlier than deep layer II cells. This delay is probably underestimated based on the stronger hyperpolarizing effect of baclofen on SL cells (Suzuki and Bekkers, 2011). Under stimulus conditions presented here, the earlier recruitment of superficial cells by sensory inputs is consistent with the early predominant superficial cell activation seen in vivo with current source density analysis (Ketchum and Haberly, 1993).

\section{Discussion}

Principal cells in the aPC display unique patterns of microcircuit design. Sensory and intracortical circuits activate different cells at different proportions, depending on their position along the superficial-to-deep gradient that also correlates with input resistance and burstiness of spiking. LSPS experiments show that intracortical connectivity gradually increases with the principal cell's position on the vertical axis from superficial to deep layers (Fig. $7 B-D$ ), a higher burstiness, and a lower input resistance. Fast two-photon population $\mathrm{Ca}^{2+}$ imaging of AP-mediated somatic $\mathrm{Ca}^{2+}$ transients in layer II evoked by selective layer Ia stimulation indicates stronger sensory recruitment of L2Ss (Fig. 7A, gray connection).

\section{Limitations}

When interpreting connectivity studies in acute brain slice preparations using LSPS, one has to consider that the analysis is biased toward local axonal projections preserved in the slicing plane (Steriade, 2001). Morphological reconstructions of L2P cell axons did not reveal a predominant local projection pattern in the anteroposterior axis as opposed to the dorsoventral axis (Johnson et al., 2000). We therefore chose the coronal slicing plane to analyze cell-type-specific local intracortical circuitry in the dorsoventral axis.

The limitations of the connectivity coefficient as a layerspecific measure of connectivity also needs to be discussed. Different source cell types in different cortical layers display distinct response patterns to LSPS and cannot be segregated. LSPS cannot resolve the single presynaptic cell types in layer II. Single-cell resolution connectivity mapping can be achieved using $\mathrm{Ca}^{2+}$ imaging-assisted identification of presynaptic cells selectively activated with 2P uncaging (Nikolenko et al., 2007) or rabies virusdependent retrograde mono-trans-synaptic labeling (Miyamichi et al., 2010).

Glutamate uncaging results in a cell-type-dependent amount of spatially adjacent suprathreshold hot spots that represent a single presynaptic cell. This makes it difficult to extract the specific stochiometry of presynaptic source and postsynaptic target cells (but see Bendels et al., 2010). We assume that LSPS samples identical populations of (presynaptic) source cells in a given layer

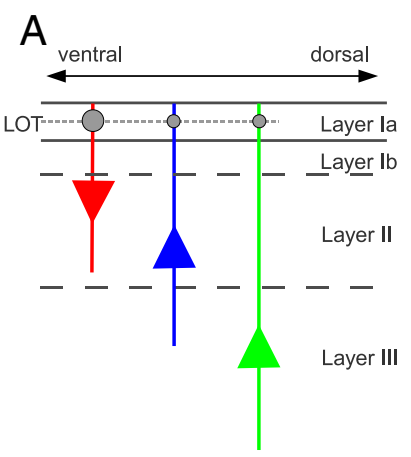

B
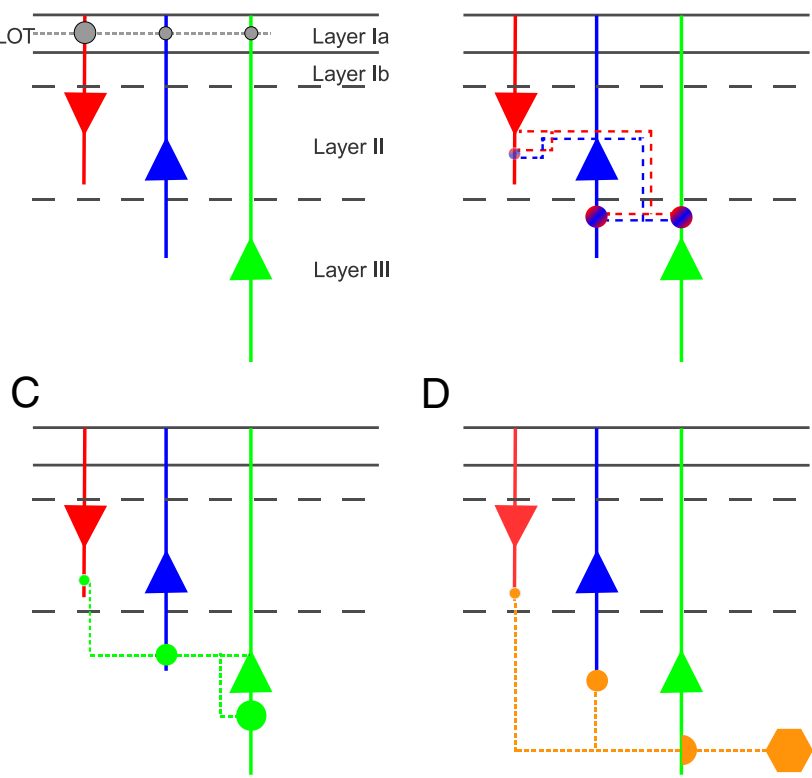

$\mathrm{D}$

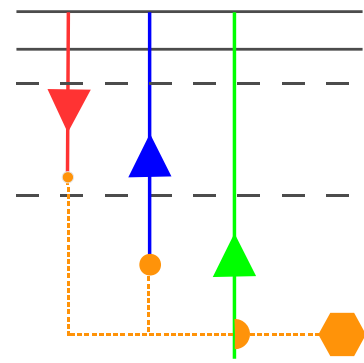

Figure 7. Circuit diagram of aPC sensory and intracortical excitatory microcircuits. Organization of an excitatory microcircuit in the aPC is shown. $\boldsymbol{A}$, Sensory input via the LOT (gray dashed line) is projected to the L2S (red downward triangle), L2P (blue upward triangle), and L3P (green upward triangle) via synapses (gray discs) in layer la. The radius of the discs indicates the input strength. The colored vertical bars indicate the dendrites of the respective cell types. $\boldsymbol{B}-\boldsymbol{D}$, The strength of the intracortical connections (colored discs) is arranged in a superficial to deep gradient complementing sensory input strength. Colored dashed lines thereby indicate the axons. Note that LSPS does not allow us to distinguish between semilunar and layer I pyramidal cell inputs ( $\boldsymbol{B}$, red and blue circles). The intracortical projection strength from deep layer III multipolar cells ( $\boldsymbol{D}$, orange) follows the same gradient as the L2S/L2P projections (blue, red) and the L3P projections ( $\boldsymbol{C}$, green). The strong synaptic pathway from those multipolar cells to the L3P cells exhibits a spatial asymmetry ( $\boldsymbol{D}$, orange semicircle) in that most of these projections arise from multipolar cells located more dorsally than the L3P.

and thus focus quantification of microcircuitry on layer-specific differences in the input pattern of identified target cells.

Intracortical connections along the dorsoventral axis: asymmetry and differential targeting by multipolar cells

The aPC has been divided into different subregions with unknown functional differences along the dorsoventral axis (Ekstrand et al., 2001). On this axis, L3P inputs display a significant asymmetric dorsal shift of their deep layer III inputs (Fig. 7D). aPC inhibitory inputs to pyramidal cells are also asymmetrically organized with a posterior shift on the anteroposterior axis (Luna and Pettit, 2010). The concept of layer-specific asymmetric local excitatory connections from deeper layers has recently been the focus of studies in auditory (Oviedo et al., 2010) and medial entorhinal (Beed et al., 2010) cortices, with interesting computational implications in these areas. In layer III, there is a superficial to deep shift of phenotype from pyramidal to multipolar cells (Neville and Haberly, 2004). It is tempting to speculate that the observed asymmetry is attributable to specific projection patterns of multipolar cells onto L3Ps (see also Fig. 7D, asymmetric orange connection).

The organization of intracortical and sensory circuits in aPC layer II can be described as a converse gradient

A recent publication by Suzuki and Bekkers (2011) compared synaptic properties of preselected canonical L2S and L2P cells. 
Their results complement the findings from LSPS-mediated connectivity analysis and population $\mathrm{Ca}^{2+}$ imaging presented here. $\mathrm{L} 2 \mathrm{P}$ cells were shown to have strong layer $1 \mathrm{~b}$ inputs, matching the increase in intracortical connectivity we observed when moving deeper in layer II. L2S cells have strong la inputs and weak 1b inputs, consistent with their low intracortical connectivity found in the present study. The more effective recruitment of superficial layer II cells by sensory activation we observed on the population level reflects the larger input strength of sensory synapses onto these cells.

When using cluster analysis of 22 intrinsic and morphological parameters, Suzuki and Bekkers (2011) drew the conclusion that layer II cells fall into two distinct groups. The functional analysis presented is then limited to a preselected subpopulation of canonical SL and SP cells (Suzuki and Bekkers, 2011). Our clustering approach operates on a much smaller number (3) of phenotypical parameters (including position). We thus cannot make statements about the distinctness of two cell groups with respect to functional microcircuit organization. We, rather, use clustering as a tool for aiding subsequent data analysis. Note that our cluster analysis generating two groups does neither confirm nor disprove the findings of Suzuki and Bekkers (2011). In their discussion, Suzuki and Bekkers also hinted at the possibility that the "distinction between SL and SP-cells might not always be clear cut" (Suzuki and Bekkers, 2011). Our results are also consistent with a continuous distribution of cell phenotypes, though (Fig. 1A, $B$, scatter plots). Most notably, our connectivity data correlate with the intrinsic cellular properties (Fig. $4 B, C$ ).

We focus on the functional analysis of local associative microcircuitry of principals cells over the whole expanse of layer II and superficial layer III. This functional perspective, rather, supports the view of graded transitions of microcircuit patterns from sensory to intracortical for aPC projection neurons oriented along a vertical, superficial-to-deep axis (for a summary, see Fig. 7). However, as we have limited our analysis to a subset of factors defining a cell type, we cannot rule out with certainty that the observed functional gradient appears as a result of a superposition of several distinct cell populations.

\section{Implications for cortical odor coding}

Firing patterns in the aPC and posterior piriform cortex during behavioral tasks (Calu et al., 2007; Roesch et al., 2007; Schoenbaum and Eichenbaum, 1995), in vivo population $\mathrm{Ca}^{2+}$ imaging (Stettler and Axel, 2009), and morphometry of axonal projection patterns of neighboring layer II pyramidal cells in the PC (Johnson et al., 2000) are compatible with the characteristic features of an association cortex. As a consequence, a model of the PC as a hybrid recurrent/feedforward pattern correlation network has been put forward (Haberly, 2001). In all these approaches, piriform cortex projection neurons are viewed as a homogenous cell population embedded equally into sensory and intracortical excitatory microcircuits. Such an architecture has been shown to be specifically suited to facilitate discrimination learning (Leibold and Bendels, 2009).

From a network perspective, our results suggest that olfactory cortex principal cell microcircuitry is organized in many subcircuits, integrating various proportions of sensory and intracortical inputs. Therefore, sensory processing will be dominated by cells with intrinsic properties different from those mainly recruited by intracortical processing. An interesting parameter, in this respect, is the difference in burst index, i.e., spike frequency adaptation seen within layer II and layer III cells. For layer II cells, this difference in neuronal output mode has been speculated to play a role in olfactory coding (Suzuki and Bekkers, 2006). We propose that spike frequency adaptation of aPC projection neurons indicates incorporation into sensory and/or intracortical microcircuits. Cells receiving a high proportion of sensory content will fire with lower spike frequency adaptation rates than cells activated by intracortical circuits. So far, the view of the PC as an association cortex is mainly based on studies not taking into account the distinct connectivities and output modes of cells (Schoenbaum and Eichenbaum, 1995; Johnson et al., 2000; Haberly, 2001; Calu et al., 2007; Roesch et al., 2007; Stettler and Axel, 2009). Additional studies in vivo will have to determine whether cells with different output modes differentially encode olfactory information.

Recently, we demonstrated that bursting is a prerequisite for the induction of plasticity in associative layer $1 \mathrm{~b}$ synapses (Johenning et al., 2009). aPC cells with high burst indices also display initial bursts firing at high frequencies (Suzuki and Bekkers, 2006). As the incorporation into the local circuitry is related to a principal cell's burst index, we propose that bursting and the related spike frequency adaptation may be an intrinsic mechanism not only relevant for odor encoding but also synaptic plasticity and odor learning.

\section{References}

Ambros-Ingerson J, Granger R, Lynch G (1990) Simulation of paleocortex performs hierarchical clustering. Science 247:1344-1348.

Barkai E, Bergman RE, Horwitz G, Hasselmo ME (1994) Modulation of associative memory function in a biophysical simulation of rat piriform cortex. J Neurophysiol 72:659-677.

Beed P, Bendels MH, Wiegand HF, Leibold C, Johenning FW, Schmitz D (2010) Analysis of excitatory microcircuitry in the medial entorhinal cortex reveals cell-type-specific differences. Neuron 68:1059-1066.

Bendels MH, Beed P, Leibold C, Schmitz D, Johenning FW (2008) A novel control software that improves the experimental workflow of scanning photostimulation experiments. J Neurosci Methods 175:44-57.

Bendels MH, Beed P, Schmitz D, Johenning FW, Leibold C (2010) Detection of input sites in scanning photostimulation data based on spatial correlations. J Neurosci Methods 192:286-295.

Calu DJ, Roesch MR, Stalnaker TA, Schoenbaum G (2007) Associative encoding in posterior piriform cortex during odor discrimination and reversal learning. Cereb Cortex 17:1342-1349.

Chiovini B, Turi GF, Katona G, Kaszas A, Erdelyi F, Szabo G, Monyer H, Csakanyi A, Vizi ES, Rozsa B (2010) Enhanced dendritic action potential backpropagation in parvalbumin-positive basket cells during sharp wave activity. Neurochem Res 35:2086-2095.

De Felipe J, Jones EG (2010) Neocortical microcircuits. In: Handbook of brain microcircuits, Ed 1 (Shepherd GM, Grillner S, eds), pp 5-14. New York: Oxford UP.

Douglas RJ, Martin KAC (2010) Canonical cortical circuits. In: Handbook of brain microcircuits, Ed 1 (Shepherd GM, Grillner S, eds), pp 15-21. New York: Oxford UP.

Dunfield D, Haas K (2009) Metaplasticity governs natural experiencedriven plasticity of nascent embryonic brain circuits. Neuron 64: $240-250$.

Dunfield D, Haas K (2010) In vivo single-cell excitability probing of neuronal ensembles in the intact and awake developing Xenopus brain. Nat Protoc 5:841-848.

Ekstrand JJ, Domroese ME, Johnson DM, Feig SL, Knodel SM, Behan M, Haberly LB (2001) A new subdivision of anterior piriform cortex and associated deep nucleus with novel features of interest for olfaction and epilepsy. J Comp Neurol 434:289-307.

Franks KM, Isaacson JS (2005) Synapse-specific downregulation of NMDA receptors by early experience: a critical period for plasticity of sensory input to olfactory cortex. Neuron 47:101-114.

Haberly LB (1983) Structure of the piriform cortex of the opossum. I. Description of neuron types with Golgi methods. J Comp Neurol 213: 163-187.

Haberly LB (2001) Parallel-distributed processing in olfactory cortex: new 
insights from morphological and physiological analysis of neuroqnal circuitry. Chem Senses 26:551-576.

Haberly LB, Price JL (1978) Association and commissural fiber systems of the olfactory cortex of the rat. J Comp Neurol 178:711-740.

Johenning FW, Holthoff K (2007) Nuclear calcium signals during L-LTP induction do not predict the degree of synaptic potentiation. Cell Calcium 41:271-283.

Johenning FW, Beed PS, Trimbuch T, Bendels MH, Winterer J, Schmitz D (2009) Dendritic compartment and neuronal output mode determine pathway-specific long-term potentiation in the piriform cortex. J Neurosci 29:13649-13661.

Johnson DM, Illig KR, Behan M, Haberly LB (2000) New features of connectivity in piriform cortex visualized by intracellular injection of pyramidal cells suggest that "primary" olfactory cortex functions like "association" cortex in other sensory systems. J Neurosci 20:6974-6982.

Ketchum KL, Haberly LB (1993) Membrane currents evoked by afferent fiber stimulation in rat piriform cortex. I. Current source-density analysis. J Neurophysiol 69:248-260.

Leibold C, Bendels MH (2009) Learning to discriminate through long-term changes of dynamical synaptic transmission. Neural Comput 21: $3408-3428$.

Lorincz A, Rozsa B, Katona G, Vizi ES, Tamas G (2007) Differential distribution of NCX1 contributes to spine-dendrite compartmentalization in CA1 pyramidal cells. Proc Natl Acad Sci U S A 104:1033-1038.

Luna VM, Pettit DL (2010) Asymmetric rostro-caudal inhibition in the primary olfactory cortex. Nat Neurosci 13:533-535.

Luna VM, Schoppa NE (2008) GABAergic circuits control input-spike coupling in the piriform cortex. J Neurosci 28:8851-8859.

Miyamichi K, Amat F, Moussavi F, Wang C, Wickersham I, Wall NR, Taniguchi H, Tasic B, Huang ZJ, He Z, Callaway EM, Horowitz MA, Luo L (2010) Cortical representations of olfactory input by trans-synaptic tracing. Nature 472:191-196.

Neville KR, Haberly LB (2004) Olfactory cortex. In: The synaptic organization of the brain, Ed 5 (Shepherd GM, ed), pp 415-454. New York: Oxford UP.

Nikolenko V, Poskanzer KE, Yuste R (2007) Two-photon photostimulation and imaging of neural circuits. Nat Methods 4:943-950.

Oviedo HV, Bureau I, Svoboda K, Zador AM (2010) The functional asymmetry of auditory cortex is reflected in the organization of local cortical circuits. Nat Neurosci 13:1413-1420.

Poo C, Isaacson JS (2009) Odor representations in olfactory cortex: "sparse" coding, global inhibition, and oscillations. Neuron 62:850-861.

Roesch MR, Stalnaker TA, Schoenbaum G (2007) Associative encoding in anterior piriform cortex versus orbitofrontal cortex during odor discrimination and reversal learning. Cereb Cortex 17:643-652.

Schoenbaum G, Eichenbaum H (1995) Information coding in the rodent prefrontal cortex. I. Single-neuron activity in orbitofrontal cortex compared with that in pyriform cortex. J Neurophysiol 74:733-750.

Shepherd GM, Pologruto TA, Svoboda K (2003) Circuit analysis of experience-dependent plasticity in the developing rat barrel cortex. Neuron 38:277-289.

Smetters D, Majewska A, Yuste R (1999) Detecting action potentials in neuronal populations with calcium imaging. Methods 18:215-221.

Steriade M (2001) The intact and sliced brain. Cambridge, MA: MIT.

Stettler DD, Axel R (2009) Representations of odor in the piriform cortex. Neuron 63:854-864.

Stokes CC, Isaacson JS (2010) From dendrite to soma: dynamic routing of inhibition by complementary interneuron microcircuits in olfactory cortex. Neuron 67:452-465.

Stosiek C, Garaschuk O, Holthoff K, Konnerth A (2003) In vivo two-photon calcium imaging of neuronal networks. Proc Natl Acad Sci U S A 100: $7319-7324$.

Suzuki N, Bekkers JM (2006) Neural coding by two classes of principal cells in the mouse piriform cortex. J Neurosci 26:11938-11947.

Suzuki N, Bekkers JM (2010) Distinctive classes of GABAergic interneurons provide layer-specific phasic inhibition in the anterior piriform cortex. Cereb Cortex 20:2971-2984.

Suzuki N, Bekkers JM (2011) Two layers of synaptic processing by principal neurons in piriform cortex. J Neurosci 31:2156-2166.

Tang AC, Hasselmo ME (1994) Selective suppression of intrinsic but not afferent fiber synaptic transmission by baclofen in the piriform (olfactory) cortex. Brain Res 659:75-81.

Treves A, Rolls ET (1992) Computational constraints suggest the need for two distinct input systems to the hippocampal CA3 network. Hippocampus 2:189-199.

Tseng GF, Haberly LB (1989) Deep neurons in piriform cortex. I. Morphology and synaptically evoked responses including a unique high-amplitude paired shock facilitation. J Neurophysiol 62:369-385.

Wilson DA, Barkai E (2010) Olfactory cortex. In: Handbook of brain microcircuits, Ed 1 (Shepherd GM, Grillner S, eds), pp 263-273. New York: Oxford UP.

Yang J, Ul Quraish A, Murakami K, Ishikawa Y, Takayanagi M, Kakuta S, Kishi K (2004) Quantitative analysis of axon collaterals of single neurons in layer IIa of the piriform cortex of the guinea pig. J Comp Neurol 473:30-42. 